\title{
Control of weakly blowing up semilinear heat equations
}

\author{
by \\ Enrique FERNÁNDEZ-CARA* \\ Departamento de Ecuaciones Diferenciales y Análisis Numérico \\ Universidad de Sevilla \\ 41080 Sevilla, Spain. \\ e-mail: cara@numer.us.es \\ and \\ Enrique ZUAZUA ${ }^{\dagger}$ \\ Departamento de Matemática Aplicada \\ Universidad Complutense \\ 28040 Madrid, Spain. \\ e-mail: zuazua@eucmax.sim.ucm.es
}

\begin{abstract}
In these notes we consider a semilinear heat equation in a bounded domain of $\mathbb{R}^{d}$, with control on a subdomain and homogeneous Dirichlet boundary conditions. We consider nonlinearities for which, in the absence of control, blow up arises.

We prove that when the nonlinearity grows at infinity fast enough, due to the local (in space) nature of the blow up phenomena, the control may not avoid the blow up to occur for suitable initial data. This is done by means of localized energy estimates.

However, we also show that when the nonlinearity is weak enough, and provided the system admits a globally defined solution (for some initial data and control), the choice of a suitable control guarantees the global existence of solutions and moreover that the solution may be driven in any finite time to the globally defined solution. In order for this to be true
\end{abstract} we require the nonlinearity $f$ to satisfy at infinity the growth condition

$$
\frac{f(s)}{|s| \log ^{3 / 2}(1+|s|)} \rightarrow 0 \quad \text { as }|s| \rightarrow \infty .
$$

This is done by means of a fixed point argument and a careful analysis of the control of linearized heat equations relying on global Carleman estimates. The problem of controlling

\footnotetext{
*Supported by grant PB95-1242 of the DGICYT (Spain).

${ }^{\dagger}$ Supported by grant PB96-0663 of the DGES (Spain).
} 
the blow up in this sense remains open for nonlinearities growing at infinity like $f(s) \sim$ $|s| \log ^{p}(1+|s|)$ with $3 / 2 \leq p \leq 2$.

\section{Introduction, problem formulation and main results}

\subsection{Problem formulation}

Let $\Omega \subset \mathbb{R}^{d}$ be a bounded domain with boundary of class $C^{2}$, let $T>0$ be given and assume $f: \mathbb{R} \mapsto \mathbb{R}$ is locally Lipschitz-continuous.

We consider semilinear parabolic systems of the form

$$
\left\{\begin{array}{lll}
y_{t}-\Delta y+f(y)=v 1_{\omega} & \text { in } & \Omega \times(0, \infty) \\
y=0 & \text { on } & \partial \Omega \times(0, \infty) \\
y(x, 0)=y_{0}(x) & \text { in } & \Omega .
\end{array}\right.
$$

In (1.1), $y=y(x, t)$ is the state and $v=v(x, t)$ is a control that acts on the system through the non-empty open set $\omega \subset \Omega .1_{\omega}$ denotes the characteristic function of the set $\omega$.

We shall denote by $Q$ the cylinder $\Omega \times(0, \infty)$ and by $\Sigma$ its lateral boundary $\partial \Omega \times(0, \infty)$. When working in finite time intervals $0<t<T$ we shall also use the notation $Q=\Omega \times(0, T)$ and $\Sigma=\partial \Omega \times(0, T)$. In the sequel, $C$ denotes a generic positive constant that may depend on the various parameters of the problem. When writing, for instance, $C=C(\Omega, \omega)$ we underline that $C$ only depends on $\Omega$ and $\omega$.

We will assume that $y_{0} \in L^{2}(\Omega)$ and $v \in L^{\infty}(\omega \times(0, \infty))$.

For simplicity, we will require $f$ to satisfy

$$
\left|f^{\prime}(s)\right| \leq C\left(1+|s|^{p}\right) \quad \text { a.e., with } p \leq 1+4 / d .
$$

Under this condition, system (1.1) possesses exactly one local (in time) solution for each $y_{0}$ and $v$ as above. Moreover the following alternative holds: Either the solution may be extended to the infinite time interval $[0, \infty)$ (global existence) or the maximal existence time is finite (finite time blow up).

In accordance with the results in [CH1], under the growth condition

$$
|f(s)| \leq C(1+|s| \log (1+|s|)) \quad \forall s \in \mathbb{R},
$$

the solutions of (1.1) are globally defined. More precisely, one has

$$
y \in C\left([0, \infty) ; L^{2}(\Omega)\right) \cap L^{2}\left(0, T ; H_{0}^{1}(\Omega)\right),
$$

for all finite $T$.

This is also true if, instead of (1.3), we assume a "good-sign" condition, like the following:

$$
f(s) s \geq-C\left(1+|s|^{2}\right) \quad \forall s \in \mathbb{R} .
$$


These notes are devoted to analyze the case where blow up occurs. In other words, we do not impose any of these conditions (1.3) or (1.4) on $f$ guaranteeing global existence of solutions.

In order to formulate precisely the problem we sahll address, let us now fix a finite time horizon $0<T<\infty$ and analyze system (1.1) in the time interval $[0, T]$.

We shall assume that:

$\left(E G S_{T}\right) \quad$ System (1.1) has at least a solution globally defined in the interval $[0, T]$.

More precisely, we assume that, for a suitable initial datum $y_{0}$ and control $v$, the solution $y$ of system (1.1) is well defined in $[0, T]$. We shall denote by $y^{*}$ this globally defined solution. Of course, this solution is not necessarily unique. Thus, $y^{*}$ will generically denote any of these globally defined solutions. We shall refer to this condition as $\left(E G S_{T}\right)$, as an abbreviation for "Existence of a Global Solution in the interval $[0, T]$ ".

If this condition does not hold, every solution of (1.1) blows up in a time $\leq T$, and therefore (1.1) has not any globally defined solution in the time interval $[0, T]$. In this case, the problem we shall analyze does not make sense.

We assume that $\omega \neq \Omega$. Otherwise, the problems we shall address are trivial. Indeed, if $\omega=\Omega$, for any $y_{0}$ there exists a control $v$ such that the solution of (1.1) is globally defined in $[0, \infty)$. To see this, it is sufficient to choose $y$ as the solution of the linear heat equation with initial datum $y_{0}$, with null control and to set $v=f(y)$. Then, $y$ and $v$ satisfy (1.1). This argument allows to systematicaly reduce the control problems for system (1.1) to similar ones for the linear heat equation. Thus $\omega \neq \Omega$ will be assumed. Note that the argument above does not apply as soon as $\omega \neq \Omega$. As we shall see, the situation is then much more involved and more interesting from a practical point of view.

Roughly speaking, the question we shall address is the following: May we avoid blow up phenomena to occur anywhere in $\Omega$ by means of controls that are localized in $\omega$ ?

More precisely, the problem we shall consider is as follows:

CONTROL PROBLEM: Given $0<T<\infty$ and assuming that $\left(E G S_{T}\right)$ holds, can we find for any initial datum $y_{0}$ a control $v$ such that the solution of (1.1) is globally defined in the time interval $[0, T]$ and, if yes, may the control be found so that the solution of (1.1) and the global one $y^{*}$ coincide at time $t=T$, $i$. e. so that

$$
y(T) \equiv y^{*}(T) ?
$$

Note that the control requirement is not only that the solution of (1.1) (that could blow up for particular choices of the initial datum in the absence of control, i. e. when $v \equiv 0$ ) becomes global but also whether we are able to match the solution of (1.1) and the a priori prescribed global solution $y^{*}$. A particularly interesting case is when system (1.1) has a global solution $y^{*}$ defined for all $t \geq 0$. In this case, if the question above has a positive answer, after matching the solution of (1.1) and $y^{*}$ in a finite time $T$ and then continuing the control $v$ for (1.1) as the control $v^{*}$ associated to the global solution for $t \geq T$, we are able to show that the solution of (1.1) is also well defined for all $t \geq 0$. Thus, if the answer to the problem above is afirmative 
and system (1.1) has at least a global (defined for all $t \geq 0$ ) solution for some $y_{0}^{*}$ and $v^{*}$, then for all $y_{0}$ there exists a control $v$ such that the solution of (1.1) is also globally defined for all $t \geq 0$.

To our knowledge the problem of whether (1.1) has at least one global solution for suitable initial data and right hand side is not completely well understood. Much more is known about the case where $v \equiv 0$. At this respect we quote, in particular, the work [BCMR] in which the connections between the existence of global and stationary solutions is investigated when $v \equiv 0$. Obviously, if (1.1) admits a stationary solution for some $v^{*}=v^{*}(x)$ the condition $(E G S)_{T}$ is fulfilled for all $T$ and the above problem makes sense. There is also an extensive literature in semilinear elliptic problems providing various conditions for existence of (at least) one stationary solution. Of course, the simplest case is when

$$
f(0)=0,
$$

since then, $y^{*} \equiv 0$ is a trivial stationary (and thus global) solution with $v^{*} \equiv 0$. Other examples will be given in Section 4.1 below.

As we shall see below, when facing blow up phenomena it is natural to try to control the system as fast as possible, before blow up occurs. At this respect it is important to note that, if the condition (1.5) in a time $T^{\prime}<T$, extending the control of (1.1) by $v^{*}$ for time $t \geq T^{\prime}\left(v^{*}\right.$ being the control associated to the global trajectory $y^{*}$ ), condition (1.5) is kept for all $t \geq T^{\prime}$.

The problem of controlling the blow up we are discussing makes sense for all evolution equations in which, in the absence of control, blow up arises. However, it is important to observe that, when the velocity of propagation of the system is finite, for instance, in the context of the semilinear wave equation, one can not expect a positive answer to hold. Indeed, in those cases, one can easily build solutions that blow up in a very short time, regardless what the control is, since the blow up occurs out of the domain of influence of the region where the control is supported. (See [Z3] for a more detailed discussion). However, the problem is relevant for all systems with infinite speed of propagation. For instance, it would be rather interesting to see if similar results to those we present here are also valid for semilinear Schrödinger equations (under suitable assumptions on the geometry of the domain $\omega$ where the control acts), but, to our knowledge, this is a completely open problem.

We analyze here the particular case of a semilinear, scalar, constant coefficient heat equation, but, of course, the problem makes sense for a wider class of reaction-convection-diffusion systems with variable coefficients. Most of the results we present here can be extended to this much more general setting. However, when proving positive results, in order to take into account convective terms entering on the nonlinearity, one has to combine the Carleman inequalities from [IY] and [FZ1], and this has not been done yet.

\subsection{Main results}

Our first main result is of negative nature. Indeed, as the following result from [FZ2] shows, one may not expect an afirmative answer to the control problem above for all nonlinearities with a prescribed growth rate at infinity, except if we impose rather severe growth conditions at 
infinity. In other words, one may only expect to be able to control the blow up phenomena in the sense described above when the nonlinearity grows at infinity with a very weak superlinear growth rate.

Theorem 1.1 There exist locally Lipschitz-continuous functions $f$ satisfying

$$
|f(s)| \sim|s| \log ^{p}(1+|s|) \quad \text { as }|s| \rightarrow \infty
$$

with $p>2$, for which system (1.1) fails to be controllable for all time $T>0$.

More precisely, the exist nonlinearities satisfying the growth condition above, such that $f(0)=$ 0 and for which, whatever $T>0$ is, there exist initial data $y_{0}$ such that the solution of (1.1) blows up in time $\leq T$ for all controls $v$.

Remark 1.1 (a) The nonlinearity that Theorem 1.1 provides is such that $f(0)=0$. Thus, $y^{*} \equiv 0$ is a globally defined solution of the corresponding system with $v \equiv 0$. The control problem then makes sense but Theorem 1.2 shows that we cannot expect a positive answer for this function $f$ whatever the control time $T$ is.

(b) For the proof of Theorem 1.1, we choose $p>2$ and

$$
f(s)=\int_{0}^{|s|} \log ^{p}(1+|\sigma|) d \sigma \quad \forall s \in \mathbb{R}
$$

that obviously satisfies the growth condition (1.7). We prove a localized estimate (in $\Omega \backslash \bar{\omega}$ ) showing that the control, that acts only on $\omega$, cannot compensate the blow-up phenomena occuring in $\Omega \backslash \bar{\omega}$. Arguments of this kind are well known; see for instance O. Yu. Imanuvilov [I] and A. Fursikov and O. Yu. Imanuvilov [FI] for examples of systems with power-like nonlinearities.

The same proof applies for nonlinearities that grow at infinity faster than (1.7) with $p>2$. For instance, the result of Theorem 1.1 holds when $f(s)=|s|^{q}$ with $q>1$ or for $f(s)=e^{s}$, the typical nonlinearities addressed in the blow up literature.

Therefore, roughly speaking, one can only expect a positive answer to the control problem above in classes of nonlinearities in which growth conditions are imposed so that functions $f$ behaving like (1.7) with $p>2$ are excluded.

(c) It is important to observe that the solutions to (1.1) can blow up in the absence of control as soon as $|f(s)|$ grows at infinity as in (1.7) with $p>1$. Therefore, Theorem 1.1 does not exclude an afirmative answer to the control problem above for a whole range of nonlinear systems for which blow-up occurs. Namely, when (1.7) is satisfied with $1<p \leq 2$.

(d) The proof of this Theorem (see section 2.1 below) applies to a wide class of semilinear evolution problems.

Let us now analyze the problem of establishing conditions under which (1.1) is controllable. The following result was proved in [FZ2]: 
Theorem 1.2 Let $T>0$. Assume that condition $(E G S)_{T}$ holds. Assume that $f: \mathbb{R} \mapsto \mathbb{R}$ is locally Lipschitz-continuous, it satisfies (1.2) and

$$
\frac{f(s)}{|s| \log ^{3 / 2}(1+|s|)} \rightarrow 0 \quad \text { as }|s| \rightarrow \infty .
$$

Then (1.1) is controllable at time $T>0$. More precisely, for any $y_{0} \in L^{2}(\Omega)$ the exists $v \in$ $L^{\infty}(\Omega \times(0, T))$ such that the solution of (1.1) is globally defined in the interval $[0, T]$ and satisfies

$$
y(\cdot, T) \equiv y^{*}(\cdot, T) .
$$

Remark 1.2 Without the assumption (1.2) on $f$, the uniqueness of solutions to (1.1) for a given $v$ is not guaranteed. In these conditions, arguing as in the proof of Theorem 1.2, we could deduce the existence of a control $v$ such that system (1.1) admits at least one solution that is globally defined in $[0, T]$ and satisfies (1.5). But we shall not address this problem here (see A. Khapalov [K1] for a related discussion).

\subsection{Comments}

Theorem 1.2 asserts that system (1.1) is controllable under the growth condition (1.7) provided $p<3 / 2$. Obviously, there is a gap between Theorems 1.1 and 1.2 . In other words, we do not know whether or not system (1.1) is controllable when $f$ satisfies (1.7) with $3 / 2 \leq p \leq 2$. As we shall see, the proof we give of Theorem 1.1 does not hold for $p \leq 2$. The same can be said about the proof of Theorem 1.2 when $p \geq 3 / 2$. Thus, the case $3 / 2 \leq p \leq 2$ is an interesting open problem. This issue will be discussed in more detail in sections 2 and 3 below.

For the proof of Theorem 1.2 that we shall sketch below, we use the explicit estimates on the cost of controllability for linear systems obtained in [FZ1] and the fixed point method introduced in the context of the semilinear wave equation in [Z1] and later applied to semilinear heat equations in $[\mathrm{FPZ}],[\mathrm{F}]$ and $[\mathrm{Z2}]$. Recall that the estimates in [FZ1] were obtained by adapting the global Carleman inequalities in $[\mathrm{FI}]$. There is however an aspect of the proof of Theorem 1.2 that is worth mentioning. Indeed, since the nonlinearity is in the blow up range, the first difficulty is to find controls such that the solution is globally defined in the time interval $[0, T]$. Indeed, note that when blow up occurs, typically, the blow up time decreases as the size of the initial datum increases. Thus, whatever $T>0$ is, there are data for which the solution corresponding to $v \equiv 0$ blows up in time $t \leq T$. For this reason, in the application of the fixed point argument, the control time we use is not $T$ for all the linearized systems but we rather choose the control time depending on the size of the potential of the linearized system so that, when the potential is large, the control time is small. Roughly speaking, we control the system (1.1) in a time which may be much smaller than $T$, not allowing the blow up phenomena to occur. The motivation of this strategy is in agreement with common sense: In the presence of blow up phenomena, one has to act on the system very fast, before blow up occurs. The strategy 
of choosing short time intervals to control nonlinear problems has been used in [K2] in one-space dimension.

In [FZ2] it was also proved that, under the conditions of Theorem 1.2, the set $\{y(\cdot, T): v \in$ $\left.L^{\infty}(\omega \times(0, T))\right\}$ is dense in $L^{2}(\Omega)$ for all initial data $y_{0} \in L^{2}(\Omega)$. This means that not only the trajectory may be driven to the target $y^{*}(T)$ but also as close as we wish to any other target. However, when the target we prescribe is an unstable state or leads to blow up phenomena, if we not keep controlling the system after time $T$, the system will eventually blow up. This is why the targets that globally defined trajectories provide are the most interesting ones since one guarantees the global existence of the controlled trajectory. We shall not discuss this problem here (that is usually refered to as the approximate controllability problem).

The rest of this paper is organized as follows. In Section 2 we give a sketch of the proof of Theorem 1.1. In Section 3, we describe the main ideas of the proof of Theorem 1.2. Finally, in Section 4, we briefly discuss the assumption $(E G S)_{T}$ and other related issues.

\section{On the lack of controllability}

\subsection{Sketch of the proof of Theorem 1.1}

This section is devoted to prove Theorem 1.1. We consider the nonlinearity $f$ as in (1.7), with

$$
p>2 .
$$

The nonlinearity $f$ is convex and $f(s) s<0$ for $s<0$. On the other hand,

$$
f(s) \sim|s| \log ^{p}(1+|s|) \quad \text { as }|s| \rightarrow \infty .
$$

Therefore, $f$ is in the range of nonlinearities in which blow-up occurs in the absence of control, i.e. with $v \equiv 0$.

The proof of Theorem 1.1 is based on the fact that there are initial data which lead to blow-up before time $T$, whatever the control $v$ is.

We proceed as in $[\mathrm{FI}]$, Section I.5 (see also $[\mathrm{H}]$ for a similar argument in the context of approximate controllability). Let $\rho \in \mathcal{D}(\Omega)$ be a nonnegative function such that

$$
\rho=0 \text { in } \omega, \quad \int_{\Omega} \rho d x=1 .
$$

Let any initial data $y_{0} \in L^{2}(\Omega)$ and control $v \in L^{\infty}(\omega \times(0, T))$ be given and let $y$ be the solution to (1.1). Let us multiply by $\rho$ the equation satisfied by $y$ and let us integrate over $\Omega$. Taking into account that the control is supported by $\omega \times(0, T)$ we have

$$
\int_{\Omega} v 1_{\omega} \rho d x=0
$$


and we obtain, by integration by parts,

$$
\frac{d}{d t} \int_{\Omega} \rho y d x=\int_{\Omega} \rho(\Delta y) d x-\int_{\Omega} \rho f(y) d x=\int_{\Omega} \Delta \rho y d x-\int_{\Omega} \rho f(y) d x .
$$

Moreover, since $f$ is an even function we deduce that

$$
\frac{d}{d t}\left(-\int_{\Omega} \rho y d x\right)=-\int_{\Omega}(\Delta \rho) y d x+\int_{\Omega} \rho f(|y|) d x .
$$

Let us now introduce the convex conjugate $f^{*}$ of $f$. For the moment, we assume that

$$
\rho f^{*}(2 \Delta \rho / \rho) \in L^{1}(\Omega) .
$$

We shall return to (2.5) later on. As we shall see, condition (2.5) holds only when $f$ grows fast enough at infinity. This is why condition $p>2$ is needed for this proof to apply.

Then, from Young's inequality, we have

$$
\begin{aligned}
\left|\int_{\Omega}(\Delta \rho) y d x\right| & \leq \int_{\Omega} \rho\left|\frac{\Delta \rho}{\rho}\right||y| d x \\
& \leq \frac{1}{2} \int_{\Omega} \rho f^{*}(2|\Delta \rho| / \rho) d x+\frac{1}{2} \int_{\Omega} \rho f(|y|) d x .
\end{aligned}
$$

Let us set

$$
k:=\frac{1}{2} \int_{\Omega} \rho f^{*}(2|\Delta \rho| / \rho) d x
$$

which is finite according to (2.5). From (2.4), (2.6) and (2.7), the following is deduced:

$$
\begin{aligned}
\frac{d}{d t}\left[-\int_{\Omega} \rho y d x\right] & \geq-\left|\int_{\Omega}(\Delta \rho) y d x\right|+\int_{\Omega} \rho f(|y|) d x \\
& =-k+\frac{1}{2} \int_{\Omega} \rho f(|y|) d x .
\end{aligned}
$$

From Jensen's inequality we also have

$$
\int_{\Omega} \rho f(|y|) d x \geq f\left(\int_{\Omega} \rho|y| d x\right) .
$$

On the other hand, taking into account that $f$ is increasing on $[0, \infty)$, we know that

$$
f\left(\int_{\Omega} \rho|y| d x\right) \geq f\left(\left|\int_{\Omega} \rho y d x\right|\right)=f\left(-\int_{\Omega} \rho y d x\right) .
$$

Thus, if we set $z(t)=-\int_{\Omega} \rho(x) y(x, t) d x$ for all $t$ and $z_{0}=-\int_{\Omega} \rho(x) y_{0}(x) d x$, we find that

$$
\left\{\begin{array}{l}
z^{\prime}(t) \geq-k+\frac{1}{2} f(z(t)), \\
z(0)=z_{0} .
\end{array}\right.
$$


We are now going to use (2.8) to prove that, for appropriate initial data, $z$ blows up in finite time. More precisely, let $y_{0} \in L^{2}(\Omega)$ be such that

$$
z_{0}=-\int_{\Omega} \rho(x) y_{0}(x) d x>0, \quad f\left(z_{0}\right)>2 k
$$

and assume that $z:\left[0, T_{*}\right) \mapsto \mathbb{R}$ is a $C^{1}$ function satisfying (2.8). Let us see that $T_{*}<+\infty$. The function $z$ is nondecreasing. Furthermore, if we set

$$
G\left(z_{0} ; s\right):=\int_{z_{0}}^{s} \frac{2}{f(\sigma)-2 k} d \sigma \quad \forall s \geq z_{0},
$$

we find that

$$
\frac{d}{d t} G\left(z_{0} ; z(t)\right)=\frac{2 z^{\prime}(t)}{f(z(t))-2 k} \geq 1 \quad \forall t \in\left[0, T_{*}\right) .
$$

Notice that, in view of $(2.1)$,

$$
f(\sigma) \sim \sigma \log ^{p}(1+\sigma) \quad \text { as } \sigma \rightarrow+\infty, \text { with } p>2 .
$$

Consequently,

$$
G\left(z_{0} ;+\infty\right)=\int_{z_{0}}^{+\infty} \frac{2}{f(\sigma)-2 k} d \sigma<+\infty .
$$

Going back to (2.9), we see that

$$
G\left(z_{0} ; z(t)\right)-G\left(z_{0} ; z_{0}\right)=G\left(z_{0} ; z(t)\right) \geq t \quad \forall t \in\left[0, T_{*}\right) .
$$

Combining (2.10) and (2.11), we deduce that $z$ blows up in finite time and, therefore, $y$ blows-up in $L^{1}(\Omega)$.

In fact, we have found the following estimate for the maximal time of existence:

$$
T_{*} \leq \int_{z_{0}}^{+\infty} \frac{2}{f(\sigma)-2 k} d \sigma
$$

where $k$ is given by (2.7). Obviously, as $z_{0} \rightarrow \infty$, the blow-up time of $z$ (and, consequently, the blow-up time of $y$ in $L^{1}(\Omega)$ ) tends to zero.

This completes the proof of Theorem 1.1 (assuming that (2.5) is satisfied). Indeed, we have shown that, whatever $T>0$ is, taking $y_{0} \in L^{2}(\Omega)$ with $z_{0}=-\int_{\Omega} \rho(x) y_{0}(x) d x$ sufficiently large, the solution $y$ of $(1.1)$ is not globally defined in the time interval $[0, T]$, regardless of the choice of the control $v \in L^{\infty}(\omega \times(0, T))$.

Now, it remains to prove that, for $f$ as in (2.1) and $p>2$, there exists a nonnegative function $\rho \in \mathcal{D}(\Omega)$ satisfying $(2.2)$ and $(2.5)$.

It is easy to check that

$$
f^{*}(s) \sim p|s|^{1-1 / p} \exp \left(|s|^{1 / p}\right) \quad \text { as } s \rightarrow+\infty .
$$


More precisely,

$$
f^{*}(s)=s\left[\exp \left(s^{1 / p}\right)-1\right]-\int_{0}^{\exp \left(s^{1 / p}\right)-1} \log ^{p}(1+\sigma) d \sigma
$$

and (2.12) is easy to deduce from (2.13) applying l'Hopital's rule.

Let us prove the existence of the desired function $\rho$ taking into account the asymptotic shape of $f^{*}$.

We only discuss the one-dimensional case $(d=1)$. The same arguments apply in higher dimensions. Obviously, to show that (2.5) holds, the unique delicate point concerns the behavior of $f^{*}\left(2\left|\rho^{\prime \prime}\right| / \rho\right)$ when $\rho$ vanishes. Note that we can always choose $\rho$ with support in a closed interval $I \subset \Omega \backslash \omega, \rho$ being strictly positive in the interior of $I$. Thus, the difficulties arise only at the extremes of the interval $I$. Let us focus, for instance, on the left extreme, located at $x=0$. It is then sufficient to choose $\rho \sim \exp \left(-x^{-m}\right)$ with $m>2 /(p-2)$ (recall that $p>2$ ). Indeed, if

$$
\rho(x)=\exp \left(-x^{-m}\right),
$$

then $\rho^{\prime}(x)=m x^{-(m+1)} \exp \left(-x^{-m}\right)$, and

$$
\rho^{\prime \prime}(x)=\left[-m(m+1) x^{-(m+2)}+m^{2} x^{-(2 m+2)}\right] \exp \left(-x^{-m}\right) .
$$

Consequently

$$
\frac{\left|\rho^{\prime \prime}(x)\right|}{\rho(x)}=\left|m^{2} x^{-(2 m+2)}-m(m+1) x^{-(m+2)}\right| \sim m^{2} x^{-(2 m+2)} \quad \text { as } x \rightarrow 0^{+} .
$$

Then, according to (2.12) and (2.14):

$$
f^{*}\left(2\left|\rho^{\prime \prime}(x)\right| / \rho(x)\right) \sim p m^{2(1-1 / p)} x^{-(2 m+2)(p-1) / p} \exp \left(m^{2 / p} x^{-(2 m+2) / p}\right) \quad \text { as } x \rightarrow 0^{+} .
$$

From (2.15), we see that $\rho f^{*}\left(2\left|\rho^{\prime \prime}(x)\right| / \rho\right) \in L^{1}(\Omega)$ if and only if $m>(2 m+2) / p$ or, equivalently, $m>2 /(p-2)$, as we have chosen above. This proves our assertion.

\subsection{Comments}

Let us discuss the optimality of the negative result in Theorem 1.2. Its proof is based on a localization in space of classical energy estimates for semilinear heat equations. Therefore, the critical growth conditions are determined by the interaction between the elliptic operator $-\Delta$ and the nonlinearity $f$. For second order O.D.E.'s, the critical growth condition is

$$
f(s) \sim|s| \log ^{2}|s| .
$$

Below this critical range, blow-up and localization phenomena do not occur. On the contrary, this can happen when the nonlinear terms grow faster at infinity. This explains the need of (1.7) with $p>2$ to prove Theorem 1.1. 
It is clear that the techniques we have used in the proof of Theorem 1.1 will not serve to prove negative results for nonlinearities with slower growth rate.

In what concerns the blow-up of solutions of the uncontrolled system (1.1) with $v \equiv 0$, it is well known that, when $f(s) \sim|s| \log ^{p}|s|$ with $p>2$, the blow-up is generically of pointwise nature. However, when $p<2$, the blow-up occurs globally in the whole domain $\Omega$. The case $p=2$ makes the transition: the blow-up is "regional", i.e. it occurs in an open subset of $\Omega$ (for instance, see $[\mathrm{G}]$ and $[\mathrm{GV}])$. Obviously, the arguments we have used in the proof of Theorem 1.1 , that rely on the localization of energy estimates, are compatible with pointwise blow-up but not with regional or global blow-up. Therefore, it is not reasonable to expect the same proof to extend to the case $p \leq 2$.

A more careful analysis of the critical nonlinearity shows that, in the class of nonlinearities that grow at infinity like

$$
f(s)=|s| \log ^{2}(1+|s|) g(s),
$$

the critical growth condition for $g$ is

$$
g(s) \sim \log ^{2}\left(\log ^{2}(1+|s|)\right) .
$$

Very likely, the negative result of Theorem 1.1 still applies for nonlinearities behaving at infinity like

$$
f(s) \sim|s| \log ^{2}(1+|s|) \log ^{2}\left(\log ^{p}(1+|s|)\right)
$$

with $p>2$. But this remains to be done and, anyway, $s \log ^{2}(1+|s|)$ will always be a lower bound on the growth at infinity of the nonlinearities for which Theorem 1.1 might apply.

We refer to [CKL] for a sharp analysis of nonlinear terms behaving at infinity like iterated logarithms in the context of the exact controllability of the one-dimensional semilinear wave equation.

\section{The control result}

In this section we describe the main ingredients and ideas of the proof of Theorem 1.2.

As we mentioned above, the proof is based on a fixed point argument that we describe now. We introduce a suitable linearization of the system. We then analyze in detail the control properties of the linearized system. To do this we use linear control theory and, by duality, we reduce the problem to the obtention of suitable observability estimates. These estimates are obtained flollowing closely the developments in [FI] on global Carleman inequalities. However, to prove the existence of the fixed point for the nonlinear map we need to carefuly analyze how the control depends on the various parameters of the problem (potential, control time, etc.). This is done as in [FZ2]. In the end of this section we shall also explain why this proof does not apply when the nonlinearity $f$ grows at infinity as in (1.7) with $3 / 2 \leq p \leq 2$. 


\subsection{Observability inequalities in $L^{2}$}

As we mentioned above, we first need to carefully analyze the control properties of linear heat equations with potentials. This is typically done using duality arguments that reduce the control problem to the obtention of suitable observability estimates for the corresponding adjoint systems (see $[\mathrm{L}])$.

Therefore, let us consider the adjoint system

$$
\left\{\begin{array}{lll}
-\varphi_{t}-\Delta \varphi+a \varphi=0 & \text { in } & Q \\
\varphi=0 & \text { on } & \Sigma \\
\varphi(T)=\varphi^{0} & \text { in } & \Omega .
\end{array}\right.
$$

In (3.1), $a=a(x, t)$ is a potential. We assume $a \in L^{\infty}(Q)$ and $\varphi^{0} \in L^{2}(\Omega)$. The following result was proved in [FZ1]:

Proposition 3.1 There exists $C=C(\Omega, \omega)>0$ such that

$$
\|\varphi(0)\|_{L^{2}(\Omega)}^{2} \leq \exp \left[C\left(1+\frac{1}{T}+T\|a\|_{\infty}+\|a\|_{\infty}^{2 / 3}\right)\right] \iint_{\omega \times(0, T)}|\varphi|^{2} d x d t
$$

for any $\varphi^{0} \in L^{2}(\Omega)$ and $T>0$, with $\varphi$ being the solution of (3.1).

This is usually refered to as observability estimate. Indeed, according to inequality (3.2) one can obtain complete information on the size of the solution of (3.1) by means of measuremnts made in $\omega \times(0, T)$. This type of inequality is relevant both in Control Theory and also in Inverse Problems (see [Is]).

Note that inequality (3.2) holds for any $T>0$ and region of control $\omega$. However, in the context of the wave equation, for an inequality of this kind to be true one needs to impose geometric conditions on $\omega$ and the time $T$ needs to be large enough (see [L]).

It is also important to observe that, according to (3.2), one can estimate the solution $\varphi$ at time $t=0$. Note that due to the irreversibility and the smoothing effect of system (3.1) one can not expect to recover completely the initial datum of $\varphi$ at time $t=T$. In fact, as shown in [FZ1], estimate (3.2) is sharp in what concerns the dependence of the observability constant with respect to time. Note, in particular, that the observability constant blows up as $\exp (C / T)$ as $T \rightarrow 0$, even when the potential vanishes and we are dealing with the constant coefficient heat equation. Whether the estimate we provide on how the constant depends on the size of the potential $a$ is sharp or not is an open problem which is relevant for improving the growth condition that Theroem 1.2 provides, as we shall discuss later.

This result was proved in [FZ1] using global Carleman inequalities as in [FI]. For the sake of completeness let us now recall the fundamental Carleman inequality from [FI] and [FZ1].

First we introduce a function $\eta^{0}=\eta^{0}(x)$ such that

$$
\left\{\begin{array}{l}
\eta^{0} \in C^{2}(\bar{\Omega}), \\
\eta^{0}>0 \text { in } \Omega, \quad \eta^{0}=0 \text { on } \partial \Omega, \\
\nabla \eta^{0} \neq 0 \text { in } \overline{\Omega \backslash \omega} .
\end{array}\right.
$$


We refer to $[\mathrm{FI}]$ for the proof of the existence of a function satisfying (3.3).

Let $K_{0}>0$ be such that

$$
K_{0} \geq 5 \max _{\bar{\Omega}} \eta^{0}-6 \min _{\bar{\Omega}} \eta^{0}
$$

and set

$$
\beta^{0}=\eta^{0}+K_{0}, \quad \bar{\beta}=\frac{5}{4} \max _{\bar{\Omega}} \beta^{0}, \quad \rho^{1}(x)=e^{\lambda \bar{\beta}}-e^{\lambda \beta^{0}}
$$

where $\lambda$ is a sufficiently large positive constant that only depends on $\Omega$ and $\omega$ and will be fixed later on. Notice that $\rho^{1}>0$ in $\Omega$. We also introduce

$$
\phi(x, t)=\rho^{1}(x) /[t(T-t)], \quad \rho(x, t)=\exp \left[\rho^{1}(x) /[t(T-t)]\right]=\exp (\phi(x, t))
$$

and the space

$$
Z=\left\{q \in C^{2}(\bar{Q}) ; q=0 \text { on } \Sigma\right\}
$$

The following holds:

Proposition 3.2 ([FI], [FZ1]). There exist positive constants $C_{*}, s_{1}>0$ such that

$$
\left\{\begin{array}{l}
s^{3} \int_{Q} \rho^{-2 s} t^{-3}(T-t)^{-3}|q|^{2} d x d t \\
\quad \leq C_{*}\left[\int_{Q} \rho^{-2 s}\left|\partial_{t} q+\Delta q\right|^{2} d x d t+s^{3} \int_{\omega \times(0, T)} \rho^{-2 s} t^{-3}(T-t)^{-3}|q|^{2} d x d t\right]
\end{array}\right.
$$

for all $q \in Z$ and $s \geq s_{1}$. Moreover, $C_{*}$ depends only on $\Omega$ and $\omega$ and $s_{1}$ is of the form

$$
s_{1}=\sigma_{1}(\Omega, \omega)\left(T+T^{2}\right)
$$

where $\sigma_{1}(\Omega, \omega)$ is a positive constant that only depends on $\Omega$ and $\omega$.

The observability inequality of Proposition 3.2 can be obtained applying this Carleman inequality to $q=\varphi$. We refer to [FZ1] for the details of the proof.

It is easy however to see how the exponential observability constant in (3.2) appears. Indeed, note that when $\varphi$ satisfies (3.1) the term $\partial_{t} q+\Delta q$ on the right hand side of (3.7) is replaced by $a \varphi$. In order to absorve the corresponding integral on the left hand side it is natural to choose $s$ large enough so that $s^{3} \sim\|a\|_{\infty}^{2}$ when $a$ is large. Once this is done, getting lower (resp. upper) bounds on the weights arising in the integrals on the left (resp. right) hand side of inequality (3.7), one obtains (3.2).

Let us now return to the proof of Theorem 1.1. In order to analyze the nonlinear control problem it is convenient to work with bounded (and not only $L^{2}$ ) controls. Indeed, applying inequality (3.2) one only gets $L^{2}$-controls. However, to get bounded controls we need to refine the observability estimate that (3.2) provides. This is done in the next section. 


\subsection{A refined observability inequality}

The following holds:

Proposition 3.3 There exists $C=C(\Omega, \omega)>0$ such that

$$
\|\varphi(0)\|_{L^{2}(\Omega)}^{2} \leq \exp \left[C\left(1+\frac{1}{T}+T+\left(T^{1 / 2}+T\right)\|a\|_{\infty}+\|a\|_{\infty}^{2 / 3}\right)\right]\left(\iint_{\omega \times(0, T)}|\varphi| d x d t\right)^{2}
$$

for any $\varphi^{0} \in L^{2}(\Omega)$ and $T>0$.

In inequality (3.9) the $L^{2}$ observation of Proposition 3.1 has been replaced by a $L^{1}$ observation. Note that Proposition 3.3 may be proved combining the inequality that Proposition 3.1 provides and the classical regularizing properties of the heat equation. It is alo worth mentioning that the observability constant changes but the factor $\|a\|_{\infty}^{2 / 3}$ in the exponential remains unchanged.

\subsection{Controllability of the linear heat equation with a potential}

As we mentioned above, one of the main ingredients of the proof of Theorem 1.1 is to obtain explicit estimates of the norms of the controls needed to achieve the controllability of the linear heat equation with a potential.

Thus, let us consider the system:

$$
\left\{\begin{array}{lll}
y_{t}-\Delta y+a y=v 1_{\omega} & \text { in } & Q \\
y=0 & \text { on } & \Sigma \\
y(x, 0)=y_{0}(x) & \text { in } & \Omega
\end{array}\right.
$$

where $a \in L^{\infty}(Q)$. As a consequence of Proposition 3.2, the following holds:

Theorem 3.1 For any $T>0$, any $a \in L^{\infty}(Q)$ and any $y_{0} \in L^{2}(\Omega)$ there exist controls $v \in$ $L^{\infty}(\omega \times(0, T))$ such that the corresponding solution of (3.10) satisfies

$$
y(x, T) \equiv 0 \text { in } \Omega .
$$

Furthermore, $v$ can be chosen such that the following estimate holds:

$$
\|v\|_{L^{\infty}(\omega \times(0, T))} \leq \exp \left[C\left(1+\frac{1}{T}+T+\left(T^{1 / 2}+T\right)\|a\|_{\infty}+\|a\|_{\infty}^{2 / 3}\right)\right]\left\|y_{0}\right\|_{L^{2}(\Omega)}
$$

where $C$ depends only on $\Omega$ and $\omega$.

Proof. Theorem 3.1 can be easily proved from Proposition 3.3 using a duality argument that is classical in Control Theory. 
Let us fix $T>0, a \in L^{\infty}(Q)$ and $y_{0} \in L^{2}(\Omega)$. For any $\varepsilon>0$, we consider the following functional:

$$
J_{\varepsilon}\left(\varphi^{0}\right)=\frac{1}{2}\left[\iint_{\omega \times(0, T)}|\varphi| d x d t\right]^{2}+\varepsilon\left\|\varphi^{0}\right\|_{L^{2}(\Omega)}+\int_{\Omega} \varphi(x, 0) y_{0}(x) d x \quad \forall \varphi^{0} \in L^{2}(\Omega) .
$$

Here, for each $\varphi^{0} \in L^{2}(\Omega), \varphi$ is the corresponding solution of (3.1).

It is not difficult to see that $\varphi^{0} \mapsto J_{\varepsilon}\left(\varphi^{0}\right)$ is a continuous and strictly convex function on $L^{2}(\Omega)$. Moreover, $J_{\varepsilon}$ is coercive. In fact, proceeding as in [FPZ], it can be checked that

$$
\liminf _{\left\|\varphi^{0}\right\|_{L^{2}(\Omega)} \rightarrow \infty} \frac{J_{\varepsilon}\left(\varphi^{0}\right)}{\left\|\varphi^{0}\right\|_{L^{2}(\Omega)}} \geq \varepsilon
$$

Therefore, $J_{\varepsilon}$ achieves its minimum at a unique $\widehat{\varphi}_{\varepsilon}^{0} \in L^{2}(\Omega)$. Let $\widehat{\varphi}_{\varepsilon}$ be the corresponding solution of (3.1). Arguing as in [FPZ], it is easy to see that, for some

$$
v_{\varepsilon} \in\left(\iint_{\omega \times(0, T)}\left|\widehat{\varphi}_{\varepsilon}\right| d x d t\right) \operatorname{sgn}\left(\widehat{\varphi}_{\varepsilon}\right)
$$

the solution $y_{\varepsilon}$ of $(3.10)$ satisfies

$$
\left\|y_{\varepsilon}(\cdot, T)\right\|_{L^{2}(\Omega)} \leq \varepsilon
$$

At this level one only requires the following unique continuation result to hold for the solutions of the adjoint system (3.1): If $\varphi \equiv 0$ in $\omega \times(0, T)$, then $\varphi \equiv 0$ everywhere. However, to get explicit bounds on the size of the control we need to use the observablity inequalities above.

We claim that, as a consequence of $(3.9)$, for a suitable $C=C(\Omega, \omega)>0$, we have

$$
\left\|v_{\varepsilon}\right\|_{L^{\infty}(\omega \times(0, T))} \leq \exp \left[C\left(1+\frac{1}{T}+T+\left(T^{1 / 2}+T\right)\|a\|_{\infty}+\|a\|_{\infty}^{2 / 3}\right)\right]\left\|y_{0}\right\|_{L^{2}(\Omega)} .
$$

Indeed, at the minimum $\widehat{\varphi}_{\varepsilon}^{0}$, we have $J_{\varepsilon}\left(\widehat{\varphi}_{\varepsilon}^{0}\right) \leq J_{\varepsilon}(0)=0$. Thus

$$
\frac{1}{2}\left[\iint_{\omega \times(0, T)}\left|\widehat{\varphi}_{\varepsilon}\right| d x d t\right]^{2} \leq \int_{\Omega} \widehat{\varphi}_{\varepsilon}(x, 0) y_{0}(x) d x \leq\left\|\widehat{\varphi}_{\varepsilon}(\cdot, 0)\right\|_{L^{2}(\Omega)}\left\|y_{0}\right\|_{L^{2}(\Omega)}
$$

and, as a consequence of $(3.9)$,

$$
\iint_{\omega \times(0, T)}\left|\widehat{\varphi}_{\varepsilon}\right| d x d t \leq \exp \left[C\left(1+\frac{1}{T}+T+\left(T^{1 / 2}+T\right)\|a\|_{\infty}+\|a\|_{\infty}^{2 / 3}\right)\right]\left\|y_{0}\right\|_{L^{2}(\Omega)} .
$$

From (3.16) we obtain (3.15) taking into account that

$$
\left\|v_{\varepsilon}\right\|_{L^{\infty}(\omega \times(0, T))}=\iint_{\omega \times(0, T)}\left|\widehat{\varphi}_{\varepsilon}\right| d x d t .
$$

In view of the uniform bound (3.15), by extracting an appropriate subsequence, we deduce that

$$
v_{\varepsilon} \rightarrow v \quad \text { weakly-* in } L^{\infty}(\omega \times(0, T)),
$$

where $v$ satisfies (3.12). Since we have (3.14) for all $\varepsilon>0$, we deduce that $v$ is such that the solution of (3.10) satisfies (3.11). This ends the proof. 
As an immediate consequence of Theorem 3.1 one can also estimate the control needed to drive an initial datum $y_{0}$ to a final state $z(x, T), z$ being the solution of (3.13) corresponding to an initial datum $z_{0}$ and right hand side $w$. Indeed, using the change of variable $p=y-z$, driving $y$ to $z(x, T)$ at time $t=T$ by means of the control $v$ is equivalent to driving $p$ to zero starting from $y_{0}-z_{0}$ by means of a control $\nu$. Thus, according to Theorem 3.1,

$$
\|\nu\|_{L^{\infty}(\omega \times(0, T))} \leq \exp \left[C\left(1+\frac{1}{T}+T+\left(T^{1 / 2}+T\right)\|a\|_{\infty}+\|a\|_{\infty}^{2 / 3}\right)\right]\left\|y_{0}-z_{0}\right\|_{L^{2}(\Omega)},
$$

and consequently

$\|v\|_{L^{\infty}(\omega \times(0, T))} \leq \exp \left[C\left(1+\frac{1}{T}+T+\left(T^{1 / 2}+T\right)\|a\|_{\infty}+\|a\|_{\infty}^{2 / 3}\right)\right]\left\|y_{0}-z_{0}\right\|_{L^{2}(\Omega)}+\|w\|_{L^{\infty}(\omega \times(0, T))}$.

\subsection{The fixed point method: Conclusion}

For simplicity we assume that $f(0)=0$ so that $y^{*} \equiv 0$ is a stationary solution corresponding to $v^{*} \equiv 0$.

We also assume that, for instance, $y_{0} \in C^{0, \beta}(\bar{\Omega})$ for some $\beta \in(0,1)$ and that $f$ is $C^{1}$ in a neighborhood of the origin. We set

$$
g(s)=\left\{\begin{array}{cc}
\frac{f(s)}{s} & \text { for } s \neq 0 \\
f^{\prime}(0) & \text { at } s=0
\end{array}\right.
$$

Then $g$ is continuous. According to (1.9), we also have

$$
\frac{g(s)}{\log ^{3 / 2}(1+|s|)} \rightarrow 0 \quad \text { as }|s| \rightarrow \infty
$$

Given $R>0$ we will use the truncation function $T_{R}$, which is given as follows:

$$
T_{R}(s)= \begin{cases}s & \text { if }|s| \leq R \\ R \operatorname{sgn}(s) & \text { otherwise }\end{cases}
$$

Let $z \in L^{\infty}(Q)$ be given and consider the linear system

$$
\left\{\begin{array}{lll}
y_{t}-\Delta y+g\left(T_{R}(z)\right) y=v 1_{\omega} & \text { in } & Q \\
y=0 & \text { on } & \Sigma \\
y(x, 0)=y_{0} & \text { on } & \Omega .
\end{array}\right.
$$


Obviously, (3.20) is of the form (3.10), with $a=g\left(T_{R}(z)\right) \in L^{\infty}(Q)$. Note that one can get an uniform bound for all these potentials $a$ depoending only on $R>0$. Let us set

$$
T_{z}^{*}=\min \left[T,\left\|g\left(T_{R}(z)\right)\right\|_{\infty}^{-2 / 3},\left\|g\left(T_{R}(z)\right)\right\|_{\infty}^{-1 / 3}\right] .
$$

According to Theorem 3.1, there exist controls $v_{z} \in L^{\infty}\left(\omega \times\left(0, T_{z}^{*}\right)\right)$ such that the solution of (3.20) in $\Omega \times\left(0, T_{z}^{*}\right)$ with $v=v_{z}$ satisfies $y\left(x, T_{z}^{*}\right)=0$ in $\Omega$ and

$$
\left\|v_{z}\right\|_{L^{\infty}\left(\omega \times\left(0, T_{z}^{*}\right)\right)} \leq C_{0}\left(\Omega, \omega, T_{z}^{*},\left\|g\left(T_{R}(z)\right)\right\|_{\infty}\right)\left\|y_{0}\right\|_{L^{2}(\Omega)},
$$

where

$$
C_{0}\left(\Omega, \omega, T_{z}^{*},\left\|g\left(T_{R}(z)\right)\right\|_{\infty}\right)=e^{C\left(1+\frac{1}{T_{z}^{*}}+T_{z}^{*}+\left(\left(T_{z}^{*}\right)^{1 / 2}+T_{z}^{*}\right)\left\|g\left(T_{R}(z)\right)\right\|_{\infty}+\left\|g\left(T_{R}(z)\right)\right\|_{\infty}^{2 / 3}\right)} .
$$

Let $A(z) \subset L^{\infty}(\omega \times(0, T))$ be the family formed by the extensions by zero to the whole interval $[0, T]$ of all these controls. Assume that $v_{z} \in A(z)$. From (3.21) and (3.22)-(3.23), it is clear that

$$
\left\|v_{z}\right\|_{L^{\infty}(\omega \times(0, T))} \leq \exp \left[C\left(1+\left\|g\left(T_{R}(z)\right)\right\|_{\infty}^{2 / 3}\right)\right]\left\|y_{0}\right\|_{L^{2}(\Omega)},
$$

where $C=C(\Omega, \omega, T)$.

Now, let $\Lambda(z) \subset L^{\infty}(Q)$ be the family of the solutions of (3.20) corresponding to the controls $v_{z} \in A(z)$. Obviously, if $y_{z} \in \Lambda(z)$, one has

$$
y \equiv 0 \quad \text { in } \Omega \times\left(T_{z}^{*}, T\right) .
$$

In particular,

$$
y(x, T)=0 \quad \text { in } \Omega .
$$

In this way, we have introduced a set-valued mapping $z \mapsto \Lambda(z)$ which depends on $R$. The proof of Theorem 1.2 will be achieved if we check that, for some $R$, this mapping possesses at least one fixed point $y$ such that

$$
\|y\|_{\infty} \leq R
$$

To this end, we apply Kakutani's Theorem to $\Lambda$. Indeed, it is not difficult to see that $\Lambda(z)$ is, for each $z \in L^{\infty}(Q)$, a nonempty closed convex set. From parabolic regularity, we also see that there exists a fixed compact subset $K \subset L^{\infty}(Q)$ such that $\Lambda(z) \subset K$ for all $z \in L^{\infty}(Q)$ (we are using here the hypothesis $\left.y_{0} \in C^{0, \beta}(\bar{\Omega})\right)$. Furthermore, $z \mapsto \Lambda(z)$ is upper hemicontinuous, i.e. the real-valued function

$$
z \mapsto \sup _{y \in \Lambda(z)}\langle\mu, y\rangle
$$

is upper semicontinuous for each bounded linear form $\mu$ on $L^{\infty}(Q)$. Consequently, it is sufficient to show that $\Lambda\left(B_{R}\right) \subset B_{R}$ if $R>0$ is large enough.

Let $y$ be a solution of (3.20) associated to $z$ and the control $v_{z}$. From classical $L^{\infty}$ estimates on the solutions of (3.20), we have

$$
\|y\|_{\infty} \leq e^{\left\|g\left(T_{R}(z)\right)\right\|_{\infty} T_{z}^{*}}\left\|y_{0}\right\|_{L^{\infty}(\Omega)}+T_{z}^{*} e^{\left\|g\left(T_{R}(z)\right)\right\|_{\infty} T_{z}^{*}}\left\|v_{z}\right\|_{L^{\infty}(\omega \times(0, T))} .
$$


On the other hand, in view of (3.19), for each $\eta>0$ there exists $C_{\eta}>0$ such that

$$
|g(s)|^{2 / 3} \leq C_{\eta}+\eta \log (1+|s|) \quad \forall s \in \mathbb{R} .
$$

Consequently, taking into account the definition (3.21) of $T_{z}^{*}$ and using (3.24), we deduce that

$$
\begin{aligned}
\|y\|_{\infty} & \leq e^{C\left(1+\left\|g\left(T_{R}(z)\right)\right\|_{\infty}^{2 / 3}\right)}\left(\left\|y_{0}\right\|_{\infty}+\left\|v_{z}\right\|_{\infty}\right) \\
& \leq e^{C\left(1+C_{\eta}+\eta \log \left\|T_{R}(z)\right\|_{\infty}\right)\left\|y_{0}\right\|_{\infty}} \\
& =e^{C\left(1+C_{\eta}\right)}\left\|y_{0}\right\|_{\infty}\left\|T_{R}(z)\right\|_{\infty}^{\eta C} \\
& \leq e^{C\left(1+C_{\eta}\right)}\left\|y_{0}\right\|_{\infty} R^{\eta C}
\end{aligned}
$$

where (again) $C=C(\Omega, \omega, T)$. Taking $\eta>0$ small enough to have $\eta C<1$, we deduce the existence of $R>0$ such that $\|y\|_{\infty} \leq R$ for all $z$. This completes the proof when $y_{0} \in C^{0, \beta}(\bar{\Omega})$ and $f$ is $C^{1}$ in a neighborhood of the origin.

For the detailed proof in the general case we refer to [FZ2].

\subsection{Comments}

Let us now discuss the optimality of the positive result in Theorem 1.2. The need of the growth condition (1.9) is clear when analyzing the proof of Theorem 1.2. It is a consequence of the presence of the term

$$
\exp \left(C\|a\|_{\infty}^{2 / 3}\right)
$$

in the estimate of the cost of null controllability of linear heat equations.

Roughly speaking, if the estimate of the cost of controllability contains a factor of the form $h\left(\|a\|_{\infty}\right)$, the growth of nonlinearities of order $s h^{-1}(s)$ is critical. This explains the need of the growth condition (1.9). Note that the estimate on the cost of null controllability for the linear heat equation with potential also contains a factor of the order of

$$
\exp \left(C\left(T^{1 / 2}+T\right)\|a\|_{\infty}\right) .
$$

According to this, the critical growth for $f$ would be $f(s) \sim s \log (1+|s|)$. However, we have been able to go beyond this level and reach the growth conditions (1.9) by choosing a sufficiently small effective time of control so that both quantities (3.27) and (3.28) are of the same order.

The presence of the factor (3.27) in the estimate of the cost of null controllability is a consequence of the observability estimate (3.2). It arises naturally when applying Carleman inequalities. Whether or not this factor may be dropped (or relaxed) in the observability inequalities in order to improve the growth condition (1.9) is an interesting open problem.

\section{Further comments}

\subsection{On the assumption of existence of a globally defined trajectory}

Theorem 1.2 requires the existence of at least a globally (in the time interval $[0, T]$ ) defined solution for a suitable initial datum and control, i. e. $(E G S)_{T}$ to hold. 
Obviously, this assumption is necessary since, otherwise, no global (in the time interval $[0, T]$ ) solution exists and the control problem we have addressed does not make sense.

The existence of global and/or stationary solutions for semilinear parabolic problems when $v \equiv 0$ has been the object of intensive research. See for instance the bibliography of this paper and the references of these articles. However, the wider issue of whether global solutions exist for suitable right hand sides $v$ localized in a given subdomain $\omega$ seems to be much more open.

Of course it would be interesting to have sharp sufficient conditions guaranteeing the existence of at least a globally defined solution for system (1.1), with possibly $v \neq 0$.

In fact, we do not know of any example of nonlinearity $f$ for which whatever the initial datum and the control is, all solutions of (1.1) blow up in a uniform time.

As we said above, when $f(0)=0,(E G S)_{T}$ is immediately fulfilled for all $T>0$ since $y^{*} \equiv 0$ is a stationary solution. If $f(a)=0$ for some $a \in \mathbb{R}, a \neq 0$, one can also build easily stationary solutions for suitable controls $v$ provided $\omega$ is a neighborhood of the boundary of $\Omega$. Indeed, it is then sufficient to take $y^{*} \equiv \varphi$, where $\varphi$ is of class $C^{2}$, vanishes on the boundary of $\Omega$ and takes the value $a$ in $\Omega \backslash \omega$. The corresponding control $v^{*}$ is then $v^{*}=-\Delta \varphi+f(\varphi)$.

\subsection{Extensions}

The results we have proved may be extended to other situations including boundary controls, parabolic operators with variable smooth coefficients, initial data in $L^{p}$ with $p$ not neccesarily equal to 2 , etc.

\section{References}

[B] V. Barbu, Exact controllability of the superlinear heat equation, preprint, Applied Math. Optimization, 42 (1) (2000), 73-89.

[BCMR] H. Brezis, Th. Cazenave, Y. Martel and A. Ramiandrisoa, Blow up for $u_{t}-\Delta u=g(u)$ revisited, Adv. Diff. Eqs., 1 (1) (1996), 73-90.

[CKL] P. M. Cannarsa, V. Komornik and P. Loreti, Well posedness and control of the semilinear wave equation with iterated logarithms, ESAIM:COCV, 4 (1999), 37-56.

[CH1] T. Cazenave and A. Haraux, Equations d'évolution avec nonlinéarité logarithmique, Ann. Fac. Sci. Toulouse, 2 (1980), 21-51.

[CH2] T. Cazenave and A. Haraux, Introduction aux problèmes d'évolution semilinéaires, Mathématiques \& Applications, Ellipses, Paris 1989.

[FPZ] C. Fabre, J.P. Puel and E. Zuazua, Approximate controllability of the semilinear heat equation, Proc. Royal Soc. Edinburgh, 125A (1995), 31-61. 
[F] E. Fernández-Cara, Null controllability of the semilinear heat equation, ESAIM: COCV, 2 (1997), 87-107.

[FZ1] E. Fernández-Cara and E. Zuazua, The cost of approximate controllability for heat equations: The linear case, Adv. Diff. Eqs., 5 (4-6) (2000), 465-514.

[FZ2] E. Fernández-Cara and E. Zuazua, Null and approximate controllability for weakly blowing up semilinear heat equations, Annales de l'IHP. Analyse non linéaire, 17 (5) (2000), 583616.

[FI] A. Fursikov and O. Yu. Imanuvilov, Controllability of Evolution Equations, Lecture Notes \#34, Seoul National University, Korea, 1996.

[G] V. Galaktionov, On blow-up and degeneracy for the semilinear heat equation with source, Proc. Royal Soc. Edinburgh, 115A, (1990), 19-24.

[GV] V. Galaktionov and J.L. Vázquez, Regional blow-up in a semilinear heat equation with convergence to a Hamilton-Jacobi equation, SIAM J. Math. Anal., 24 (5) (1993), 12541276.

[H] J. Henry, Contrôle d'un réacteur enzymatique à l'aide de modèles à paramètres distribués: Quelques problèmes de contrôlabilité de systèmes paraboliques, $\mathrm{Ph}$. D. Thesis, Université Paris VI, 1978.

[I] O. Yu. Imanuvilov, Exact boundary controllability of the parabolic equation, Russian Math. Surveys 48 (1993), 211-212.

[IY] O. Yu. Imanuvilov and M. Yamamoto, On Carleman inequalities for parabolic equations in Sobolev spaces of negative order and exact controllability for semilinear parabolic equations, Preprint \# 98-46, University of Tokyo, Grade School of Mathematics, Komobo, Tokyo, Japan, November 1998.

[Is] V. Isakov, Inverse Problems for Partial Differential Equations, Springer-Verlag, Berlin, 1998.

[K] A. Khapalov, Some aspects of the asymptotic behavior of the solutions of the semiliner heat equation and approximate controllability, J. Math. Anal. Appl., 194 (1995), 858-882.

[L] J.-L. Lions, Contrôlabilité exacte, stabilisation et perturbations de systèmes distribués. Tomes 1 \& 2. Masson, RMA 8 \& 9, Paris, 1988.

[Z1] E. Zuazua, Exact boundary controllability for the semilinear wave equation, in "Nonlinear Partial Differential Equations and their Applications", Vol. X, H. Brezis and J.L. Lions eds., Pitman, 1991, 357-391.

[Z2] E. Zuazua, Finite dimensional null-controllability of the semilinear heat equation, J. Math. Pures et Appl., 76 (1997), 237-264. 
[Z3] E. Zuazua, Exact controllability for the semilinear wave equation in one space dimension, Ann. IHP, Analyse non Linéaire, 10 (1993), 109-129.

[Z4] E. Zuazua, Some problems and results on the controllability of Partial Differential Equations, in "Proceedings of the Second European Conference of Mathematics", Budapest, July 1996, Progress in Mathematics, 169, 1998, Birkhäuser Verlag Basel/Switzerland, pp. 276-311. 